\title{
OUTBURSTS BY LOW-MASS WHITE DWARFS IN SYMBIOTIC VARIABLES
}

\author{
Edward M. Sion \\ Department of Astronomy and Astrophysics \\ Villanova University \\ Villanova, PA 19085 \\ and \\ Christian J. Ready \\ Department of Astronomy and Astrophysics \\ Villanova University \\ Villanova, PA 19085
}

\begin{abstract}
We have investigated the nature of $\mathrm{P}$ Cygni-like features in the high resolution $I U E$ spectra of the symbiotic variables BF Cyg and EG And by looking for orbital phase-dependent variations in the structure and velocity of the C IV (1550 $\AA)$ resonance doublet in 10 archival $I U E$ high-dispersion spectra of these systems and by comparing their profiles with $\mathrm{P}$ Cygni-like features seen in IUE high-dispersion spectra of other symbiotic stars. For BF Cyg, we find maximal P Cygni absorption equivalent widths and terminal velocities of $\sim 900 \mathrm{~m} \AA$ and $\sim-240 \mathrm{~km} \mathrm{~s}^{-1}$ respectively, full emission velocity widths at the $10 \%$ intensity level of $300-400 \mathrm{~km} \mathrm{~s}^{-1}$, depending on phase, $\Phi$, variations in the width of the P Cygni absorption, shortward velocity shifts of the C IV emission line in all but two orbital phases, relative to the C IV velocity at $\Phi=0.01$, and absorption sub-structure within the C IV P Cygni lines which coincide in velocity from image to image. In EG And the C IV P Cygni absorption equivalent width is, on average, $300 \mathrm{~m} \AA$ with a terminal velocity of $-217 \mathrm{~km} \mathrm{~s}^{-1}$ (which includes the systemic radial velocity contribution of $-93 \mathrm{~km} \mathrm{~s}^{-1}$ ). The presence in one image, of a strong absorption feature at He II (1640), having a precise velocity coincidence with the C IV P Cygni absorption component, suggests that the absorbing material responsible for both line features is associated with outflow and P Cygni self-absorption from the vicinity of the hot component. In both BF Cyg and EG And, the structure and behavior of the P Cygni profiles may rule out their origin in the red giant wind or in an expanding circumbinary shell. Our results support the hot component wind interpretation of Mikolajewska et al. (1989). Motivated by the low-mass white dwarf shown to be present in BF Cyg (Mikolajewska et al. 1989), we have carried out quasi-static evolutionary model calculations with accretion onto a $0.55 \mathrm{M}_{\odot}$ white dwarf accreting at the rate $2 \times 10^{-8} \mathrm{M}_{\odot} \mathrm{yr}^{-1}$. The sequence of
\end{abstract}


3800 quasi-static models, the first to be reported for a low-mass hot white dwarf $\left(M_{w d}<0.8 \mathrm{M}_{\odot}\right)$ accreting at a high rate, yields an unexpected outburst behavior in response to accretion at that rate, when compared to evolutionary sequences with accretion onto higher mass white dwarfs. A series of three major thermonuclear shell flashes and one lower energy shell flash is revealed with the timescale during outburst and the inter-outburst timescale substantially shorter than expected for a $0.55 \mathrm{M}_{\odot}$ white dwarf. Preliminary results on other sequences are also reported. Implications for the observations of symbiotic systems thought to contain low-mass white dwarfs, are discussed.

\section{References}

Mikolajewska, J., Kenyon, S. J., and Mikolajewska, M. (1989). A.J. 98, 1427. 